\section{Diagnostic and interventional pancreatic endosonography}

\author{
Trikudanathan G, Vega-Peralta J, Malli A, \\ Munigala $S$, Han $Y$, Bellin $M$, et al. Diagnostic \\ performance of endoscopic ultrasound for \\ non-calcific chronic pancreatitis Based on \\ Histopathology.
}

Am J Gastroenterol. 2016;111:568-74.

While diagnosing chronic pancreatitis $(\mathrm{CP})$ in advanced stages is easy and can be made on the basis of changes in the ductal anatomy, ductal and parenchymal calcifications, and the presence of exocrine and endocrine insufficiency, the diagnosis of early CP is difficult. Endoscopic ultrasound (EUS) is considered as a fairly sensitive and specific modality for the diagnosis of early $\mathrm{CP}$, but very few studies have previously compared its results with histopathological findings. This study was conducted in a cohort of patients with noncalcific $\mathrm{CP}(\mathrm{NCCP})$ who underwent total pancreatectomy and islet cell autotransplantation (TPIAT) for the management of refractory pain. The histopathological findings from the resected specimen were correlated with the findings of EUS done before surgery in these patients. Of all the patients who underwent TPIAT during the study, those with NCCP were identified by reviewing their computed tomography (CT) findings. To minimize the impact of interval disease progression, patients who underwent EUS within 1 year preceding surgery were only included in the analysis.

The EUS was performed by experienced endosonographer and the nine equally weighted criteria given by Wiersema et al. were used for the diagnosis of $\mathrm{CP} \cdot{ }^{[1]}$ However, the authors did not include hyperechoic duct walls in the head of pancreas as an EUS feature for diagnosis of $\mathrm{CP}$ whereas other EUS features such as cysts, dilated side branches, and hyperechoic foci and strands were included in the diagnosis of CP. A blinded pathologist scored the histological findings and fibrosis from the head, body, and tail of the pancreas obtained at the time of TPIAT as per the pathology classification system proposed by Ammann et al. ${ }^{[2]}$

A total of 68 patients with refractory pain were included in the analysis, of whom $76.5 \%$ had history of an episode of acute pancreatitis, $54 \%$ were smokers, and $62 \%$ had been alcoholic. Of 68 patients, 56 patients had abnormal histology, and 16 had evidence of severe fibrosis with median fibrosis score of 4.7. Twelve patients had normal histology. Interestingly, of these 12 patients, $2(16.7 \%)$ had 0/9 EUS features, $3(25 \%)$ had 2/9
EUS features, 5 (41.7\%) had 3/9 EUS features, and 2 (16.7\%) had 4/9 EUS features of CP.

The correlation of the fibrosis score and number of EUS criteria present was low, but statistically significant $(r=0.24$, $P=0.044)$. The presence of four or more EUS criteria had the best combination of sensitivity and specificity $(61 \%$ sensitivity and $75 \%$ specificity) for predicting abnormal histology. Of the patients with CP, 9 patients had no EUS criteria, 2 patients had one criterion, and 8 patients had 3 criteria. None of the EUS features individually had any correlation with any specific histological pattern. Only main pancreatic ductal irregularity on EUS was found to independently predictor $\mathrm{CP}$. The authors concluded that EUS correlates poorly with histopathology in NCCP and therefore should not be used alone to diagnose $\mathrm{CP}$.

\section{Commentary}

$\mathrm{CP}$ is a chronic debilitating inflammatory disorder of the pancreas characterized by irreversible damage to pancreatic parenchyma leading on to pain and endocrine as well as exocrine insufficiency. ${ }^{[1]}$ While it is easy to recognize an advanced CP, early stages characterized by the absence of calcification, ductal changes, and without endocrine and exocrine insufficiency are difficult to diagnose accurately. ${ }^{[3-5]}$ In such situations, functional tests and use of secretin-enhanced magnetic resonance cholangiopancreatography (MRCP) have been resorted to, but these tests are difficult to perform, have diagnostic limitations, and have limited availability. Although histology is considered to be the gold standard but tissue samples are rarely available from pancreas in clinical practice. EUS, because of its proximity to pancreas, provides an excellent ability to visualize the pancreatic parenchyma as well as pancreatic duct and possibly diagnose early CP. ${ }^{[6,7]}$ In this regard, various EUS-based diagnostic criteria have been proposed including the Rosemont criteria. ${ }^{[7,8]}$ While few studies have compared EUS findings with ERCP and imaging findings, only limited literature is available comparing EUS findings with histology. ${ }^{[9-12]}$ Of 42 patients in a previous study who had NCCP, the presence of 4 or more criteria provided a high sensitivity (90\%) and specificity (85.7\%), and the number of criteria present was seen to correlate with the severity of $\mathrm{CP}^{\left[{ }^{[10]}\right.}$ Another previous report had suggested that the presence of three or more criteria provided best sensitivity $(83 \%)$ and specificity (80\%) ${ }^{[11]}$ In addition, mild fibrosis is known to occur in elderly, especially who smoke or drink alcohol. ${ }^{[12]}$

Thus, the currently available evidence suggests that EUS diagnosis of $\mathrm{CP}$ is not very accurate, and there is a concern about inter-observer variability in reporting EUS findings. ${ }^{[13]}$ Moreover, the current study now suggests that even presence of more than 4 criteria do not conclusively establish the diagnosis 
of NCCP. Moreover, an interesting conclusion of the current study is that even a normal EUS cannot confidently rule out CP. This is an important message from this study and therefore there is a need to devise a new scoring system for diagnosis of NCCP that may take into account other factors such as age, sex, body mass index, and smoking and alcohol exposure along with EUS findings. However, the authors found that the presence of 6 or more EUS features had $100 \%$ specificity to diagnose NCCP. The present study has its limitations including the absence of elastography values, lack of clarity if EUS was done late enough after acute pancreatitis, detailed description of the patients who had EUS findings suggestive but did not have NCCP so as to identify the confounders as well as the uncertainty of Ammann scoring system for quantifying fibrosis. In addition, the study is retrospective, and a clear selection bias for more severe disease is likely as patients included were the surgical patients who were refractory to medical therapy. The present study emphasizes that EUS as a standalone test to diagnose NCCP is not correct, and there is a need to devise new criteria for reliable diagnosis of CP. The role of EUS elastography needs to be explored in the diagnosis of NCCP.

\section{Bang JY, Hasan MK, Navaneethan U, Sutton B, Frandah W, Siddique S, Hawes RH, Varadarajulu S. Lumen apposing metal stents for drainage of pancreatic fluid collections: When and for whom?}

Dig Endosc 2016 ahead of print. doi: 10.1111/den.12681.

This retrospective study from the United States is a casecontrol comparison of 20 patients with pancreatic fluid collections (PFC's) who underwent drainage with lumen apposing metallic stents (LAMS) with forty matched patients who underwent drainage using plastic stents. The patients had undergone EUS-guided drainage for the PFCs. While selecting controls (plastic stent), the patients were matched for the type of PFC (pseudocyst or walled off necrosis (WON), and the technique used (multigate drainage or conventional) by an independent, blinded observer. The plastic stents used were two double pigtail 7-Fr, $4 \mathrm{~cm}$ stents for controls while a single LAMS (Hot AXIOSTM Stent and Electrocautery Enhanced Delivery System; Boston Scientific Corporation, Natick, MA, USA) was used in the cases.

After therapy, stents were removed in patients with normal MPD and PFCs $<2 \mathrm{~cm}$ as documented using MRCP or CT. In patients with disconnected pancreatic duct syndrome (DPDS), the plastic stents were left in situ indefinitely. In patients with DPDS who were treated with LAMS, the LAMS were exchanged for two 7-Fr double pigtail plastic stents that were left indefinitely. Treatment success was defined as the resolution of PFC and symptoms at 8 weeks.

The two groups were comparable at baseline for demographic characters, clinical features, and characteristic of PFCs. The two groups did not differ vis-à-vis the route of drainage, need for multigate drainage, placement of nasocystic or percutaneous drain, necrosectomy, or enteral feeding requirements. The procedure duration was significantly shorter for insertion of LAMS. Interestingly, there was no significant difference in success rates (95.0 vs. $92.5 \%, P=0.99)$, need for reintervention ( 25.0 vs. $30.0 \%, P=0.77$ ), clinical adverse effects ( 10.0 vs. $12.5 \%, P=0.99)$, stent-related adverse effects ( 10.0 vs. $2.5 \%, P=0.26)$, or median length of hospital stay (2 [IQR 1-5] vs. 2 [IQR 1-7] days, $P=0.58$ ) between the two groups. The hospital cost of the two procedures was comparable in patients with WON (USD 16708 for LAMS vs. USD 17221 for plastic stents, $P=0.90$ ) whereas the mean hospital costs were significantly lower for pseudocysts using plastic stents (USD 18996 vs. USD 58649, $P=0.03$ ). Most patients requiring reintervention, as expected, had underlying WON (14/17). The authors concluded that there is no difference in clinical outcomes when treating WON with LAMS or plastic stent whereas treatment of pseudocysts is less expensive with plastic stents.

\section{Commentary}

Endoscopic drainage has emerged as the modality of choice in management of symptomatic PFCs. ${ }^{[14]}$ The drainage is usually accomplished by the creation of cystoenterostomy and placement of endoprosthesis through the tract. While EUS is clearly being preferred over direct endoscopic drainage, other factors helpful in adequate drainage are uncertain. ${ }^{[15]}$ The amount of necrotic debris (WON or pseudocyst) seems to determine the outcomes. ${ }^{[16-18]}$ With the availability of LAMS for drainage of PFCs, their role in the management of PFCs needs to be defined. Since, with time the collections containing necrotic debris tend to liquefy, it makes sense to delay the intervention till the point when the PFCs are predominantly liquid. ${ }^{[19]}$ The use of a nasojejunal feeding has been demonstrated to delay the need for intervention in these patients. ${ }^{[20]}$

The LAMS, because of their wider diameter, are presumed to be more effective in the treatment of WON. However, the results of the current study suggest that the success rates using LAMS are comparable to that using plastic stents. In addition, the stents used in plastic group were two 7-Fr stents. The use of more stents or of wider diameter (10 Fr) may have further improved results in the plastic stent group. We also have found that majority of patients with WON can be treated with multiple plastic stents with metal stents or direct necrosectomy being required in few patients only. ${ }^{[21,22]}$

In patients with DPDS long-term indwelling stents are needed to provide drainage of the pancreatic juice produced by the distal viable pancreatic parenchyma. ${ }^{[23-26]}$ Therefore, in patients with WON and DPDS, one would need additional procedure of replacing LAMS with plastic stents. This may become difficult as the cavity reduces in size significantly 
after drainage of PFC thereby creating a difficult endoscopic situation. Therefore, this paper comparing the use of LAMS and plastic stents does not clearly indicate any benefit of use of metallic stents in WON while the use of plastic stents is equi-efficacious and cost-effective in the treatment of pseudocysts.

Vishal Sharma, Surinder Singh Rana

Department of Gastroenterology, Postgraduate Institute of Medical Education and Research, Chandigarh, India

Address for correspondence: Dr. Surinder Singh Rana, Department of Gastroenterology, Postgraduate Institute of Medical Education and Research, Chandigarh - 160 012, India. E-mail: drsurinderrana@yahoo.co.in

\section{References}

1. Wiersema MJ, Hawes RH, Lehman GA, Kochman ML, Sherman S, Kopecky KK. Prospective evaluation of endoscopic ultrasonography and endoscopic retrograde cholangiopancreatography in patients with chronic abdominal pain of suspected pancreatic origin. Endoscopy 1993;25:555-64.

2. Ammann RW, Heitz PU, Klöppel G. Course of alcoholic chronic pancreatitis: A prospective clinicomorphological long-term study. Gastroenterology 1996;111:224-31.

3. Sharma V, Rana SS, Bhasin DK. Medical management of pain in chronic pancreatitis. Trop Gastroenterol 2014;35:205-11.

4. Bhasin DK, Rana SS, Chandail VS, Singh G, Gupta R, Kang M, et al. Clinical profile of calcific and noncalcific chronic pancreatitis in North India. J Clin Gastroenterol 2011;45:546-50.

5. Bhasin DK, Singh G, Rana SS, Chowdry SM, Shafiq N, Malhotra S, et al. Clinical profile of idiopathic chronic pancreatitis in North India. Clin Gastroenterol Hepatol 2009;7:594-9.

6. Gardner TB, Levy MJ. EUS diagnosis of chronic pancreatitis. Gastrointest Endosc 2010;71:1280-9.

7. Rana SS, Vilmann P. Endoscopic ultrasound features of chronic pancreatitis: A pictorial review. Endosc Ultrasound 2015;4:10-4.

8. Catalano MF, Sahai A, Levy M, Romagnuolo J, Wiersema M, Brugge W, et al. EUS-based criteria for the diagnosis of chronic pancreatitis: The Rosemont classification. Gastrointest Endosc 2009;69:1251-61.

9. Rana SS, Bhasin DK, Rao C, Singh K. Role of endoscopic ultrasound in idiopathic acute pancreatitis with negative ultrasound, computed tomography, and magnetic resonance cholangiopancreatography. Ann Gastroenterol 2012;25:133-7.

10. Varadarajulu S, Eltoum I, Tamhane A, Eloubeidi MA. Histopathologic correlates of noncalcific chronic pancreatitis by EUS: A prospective tissue characterization study. Gastrointest Endosc 2007;66:501-9.

11. Chong AK, Hawes RH, Hoffman BJ, Adams DB, Lewin DN, Romagnuolo J. Diagnostic performance of EUS for chronic pancreatitis: A comparison with histopathology. Gastrointest Endosc 2007;65:808-14.

12. Bhutani MS, Arantes VN, Verma D, Moezzi J, Suryaprasad S, Kapadia AS, et al. Histopathologic correlation of endoscopic ultrasound findings of chronic pancreatitis in human autopsies. Pancreas 2009;38:820-4.

13. Stevens T, Lopez R, Adler DG, Al-Haddad MA, Conway J, Dewitt JM, et al. Multicenter comparison of the interobserver agreement of standard EUS scoring and Rosemont classification scoring for diagnosis of chronic pancreatitis. Gastrointest Endosc 2010;71:519-26.

14. Varadarajulu S, Rana SS, Bhasin DK. Endoscopic therapy for pancreatic duct leaks and disruptions. Gastrointest Endosc Clin N Am 2013;23:863-92.
15. Sharma V, Rana SS, Bhasin DK. Endoscopic ultrasound guided interventional procedures. World J Gastrointest Endosc 2015;7:628-42.

16. Rana SS, Bhasin DK, Sharma RK, Kathiresan J, Gupta R. Do the morphological features of walled off pancreatic necrosis on endoscopic ultrasound determine the outcome of endoscopic transmural drainage? Endosc Ultrasound 2014;3:118-22.

17. Vilmann AS, Menachery J, Tang SJ, Srinivasan I, Vilmann P. Endosonography guided management of pancreatic fluid collections. World J Gastroenterol 2015;21:11842-53.

18. Bang JY, Wilcox CM, Trevino J, Ramesh J, Peter S, Hasan M, et al. Factors impacting treatment outcomes in the endoscopic management of walled-off pancreatic necrosis. J Gastroenterol Hepatol 2013;28:1725-32.

19. Rana SS, Bhasin DK, Reddy YR, Sharma V, Rao C, Sharma RK, et al. Morphological features of fluid collections on endoscopic ultrasound in acute necrotizing pancreatitis: Do they change over time? Ann Gastroenterol 2014;27:258-61.

20. Rana SS, Chaudhary V, Sharma R, Sharma V, Chhabra P, Bhasin DK. Impact of nasojejunal feeding on outcome of patients with walled off pancreatic necrosis (WOPN) presenting with pain: A pilot study. J Gastrointest Surg 2015;19:1621-4.

21. Rana SS, Bhasin DK, Rao C, Gupta R, Singh K. Non-fluoroscopic endoscopic ultrasound-guided transmural drainage of symptomatic non-bulging walled-off pancreatic necrosis. Dig Endosc 2013;25:47-52.

22. Rana SS, Sharma V, Chhabra P, Sharma R, Gupta R, Bhasin DK. Endoscopic ultrasound guided transmural drainage of walled off pancreatic necrosis using a step up approach: A single centre experience. Gastrointest Endosc 2016;83:AB340.

23. Rana SS, Bhasin DK, Rao C, Sharma R, Gupta R. Consequences of long term indwelling transmural stents in patients with walled off pancreatic necrosis \& disconnected pancreatic duct syndrome. Pancreatology 2013;13:486-90.

24. Rana SS, Bhasin DK, Sharma R, Gupta R. Factors determining recurrence of fluid collections following migration of intended long term transmural stents in patients with walled off pancreatic necrosis and disconnected pancreatic duct syndrome. Endosc Ultrasound 2015;4:208-12.

25. Arvanitakis M, Delhaye M, Bali MA, Matos C, De Maertelaer V, Le Moine $\mathrm{O}$, et al. Pancreatic-fluid collections: A randomized controlled trial regarding stent removal after endoscopic transmural drainage. Gastrointest Endosc 2007;65:609-19.

26. Varadarajulu S, Wilcox CM. Endoscopic placement of permanent indwelling transmural stents in disconnected pancreatic duct syndrome: Does benefit outweigh the risks? Gastrointest Endosc 2011;74:1408-12.

This is an open access article distributed under the terms of the Creative Commons Attribution-NonCommercial-ShareAlike 3.0 License, which allows others to remix, tweak, and build upon the work non-commercially, as long as the author is credited and the new creations are licensed under the identical terms.

\begin{tabular}{|l|c|}
\hline \multicolumn{2}{|c|}{ Access this article online } \\
Website: & Quick Response Code \\
www.jdeonline.in & \\
\hline & DOI: \\
10.4103/0976-5042.193746 & \\
\hline
\end{tabular}

How to cite this article: Sharma V, Rana SS. Diagnostic and interventional pancreatic endosonography. J Dig Endosc 2016;7:125-7. 